\title{
Documentos
}

\section{Consenso salud materna para Chile en el nuevo milenio*}

\author{
* Este texto es el resumen de las conclusiones realizadas en la Illa Reunión Bienal \\ ACOG-SOCHOG efectuada en Concepción, Chile, el 11-13 de noviembre de 2012, \\ workshop Consenso sobre Mortalidad Materna en Chile.
}

\begin{abstract}
Rogelio González $P^{* *}$ 1,8, Elard Koch C. ${ }^{2,3}$, José Andrés Poblete L. ${ }^{1}$, Claudio Vera P. ${ }^{1}$, Hernán Muñoz S.4,6,9, Guillermo Carroli 5, Edgardo Abalos 5 , André Lalonde7, Enrique Oyarzún E. ${ }^{1}$, Alfredo Germain A. ${ }^{9}$, Carlos Schnapp S. 9 , Jorge Neira M. ${ }^{1}$, Jorge Hasbun $H^{4}$, Jorge Carvajal C. ${ }^{1}$, Mónica Theodor D. ${ }^{1}$, Paula Vargas I. ${ }^{1}$, Álvaro Insunza F. 10 , Juan Kusanovic P. 1, Ricardo Gómez M.8,10, Miriam González O.11, Eghon Guzmán B. 11, Mario Paublo M. ${ }^{11}$, Soledad Burgos E. 11, Marco Clavero P. 11,12, Francisco Klassen $P .13$
\end{abstract} ${ }^{1}$ División de Obstetricia y Ginecología, Facultad de Medicina, Pontificia Universidad Católica de Chile. ${ }^{2}$ Instituto MELISA,
Epidemiología Molecular para las Ciencias de la Vida, Facultad de Medicina, Universidad Católica de la Santísima Con-
cepción, Concepción, Chile. ${ }^{3}$ Programa de Doctorado, División de Epidemiología, Escuela de Salud Pública, Facultad de
Medicina, Universidad de Chile. ${ }^{4}$ Unidad de Medicina Materno-Fetal, Hospital Clínico de la Universidad de Chile. ${ }^{5}$ Centro
Rosarino de Estudios Perinatales (CREP/OMS), Rosario, Argentina. ${ }^{6}$ Sociedad Chilena de Obstetricia y Ginecología.
${ }^{7}$ Society of Obstetricians and Gynecologists of Canada (SOGC). ${ }^{8}$ Servicio de Obstetricia y Ginecología, Clínica Santa
María, Santiago, Chile. ${ }^{9}$ Servicio de Obstetricia y Ginecología, Clínica Las Condes, Santiago, Chile. ${ }^{10}$ Servicio de Obs-
tetricia y Ginecología, Hospital Padre Hurtado, Santiago, Chile. ${ }^{11}$ Ministerio de Salud de Chile. ${ }^{12}$ Servicio de Obstetricia
y Ginecología del Hospital San José, Santiago, Chile. ${ }^{13}$ Servicio de Obstetricia y Ginecología Hospital Regional de Los
Ángeles, Los Ángeles, Chile.

${ }^{\star \star}$ Autor correspondiente de parte de integrantes de Consenso Mortalidad Materna en Chile. E-mail: roggonza@med.puc.cl

\section{RESUMEN}

Contexto: Chile presenta una tendencia secular hacia una sostenida mejoría en los principales indicadores materno-infantiles. Su situación constituye una experiencia positiva a nivel de la región de Latino-América y el Caribe. Sin embargo, esta tendencia se ha estancado en los últimos diez años lo que produce una situación inestable y preocupante desde el punto de vista de la salud pública materna. Esto motiva una reunión de expertos a nivel nacional e internacional para proponer estrategias para el alto nivel político orientadas a alcanzar los Objetivos 4 y 5 del Milenio. Conclusión: Este documento de consenso sobre mortalidad materna, sugiere un enfrentamiento en dos ejes: primero, enfrentar la nueva realidad epidemiológica desde la etapa pre-concepcional, esto incluye considerar la alta prevalencia de obesidad, hipertensión arterial, diabetes, hiperlipidemias e hipotiroidismo en la población, y por otro lado reforzar la seguridad de la asistencia del embarazo, parto y puerperio en los lugares más alejados y más vulnerables del país. Es necesario focalizar las intervenciones en los grupos de mayor riesgo vital (edades extremas de la vida fértil y portadoras de enfermedades médico-quirúrgicas severas, que se reflejan en el aumento proporcional de 
las causas indirectas de muerte materna), reforzar las actividades de auditorías de mortalidad/near miss, así como controlar el aumento alarmante de la tasa de partos por cesáreas.

\section{PALABRAS CLAVE: Mortalidad materna en Chile, consenso salud materna, objetivos del milenio (ODMs), near miss, políticas públicas materno-infantiles}

\section{SUMMARY}

Background: Chile represents a positive evolution in terms of maternal and infant health in last fifty years. This trend in last ten years has remains stable. According to this situation a National and Regional Consensus meeting about Maternal Mortality has been organized. Conclusion: Propositions have been released about how to improve maternal and child health in Chile. Main focus might be to confront the new epidemiological reality including maternal overweight/obesity, hypertension, diabetes, hyperlipidemia and hypothyroidism. Effort must be done focusing in the day around the birth, mainly in rural and poorest areas of the country. Indirect causes of maternal mortality have become the main problems in maternal care; this fact must be reflected in protocols, audits (including near miss) and public policies. High rate of cesarean section also remains a challenged.

\section{KEY WORDS: Maternal mortality in Chile, consensus in maternal health, millennium developmental goals (MDGs), near miss, public policies}

\section{Contexto Global}

En la Cumbre del Milenio de Naciones Unidas, realizada el año 2000, la comunidad internacional definió 8 grandes Objetivos de Desarrollo del Milenio (ODM), cuyas metas debieran lograrse el año 2015. Entre estos Objetivos se encuentra la reducción de la mortalidad infantil (ODM 4) y la mejoría de la salud materna (ODM 5). El logro de estos objetivos, directamente relacionados con la condición de la mujer, en particular, con su capacidad para utilizar y valorar los servicios de atención en salud, involucra avanzar también en el ODM 3, cual es el empoderamiento de la mujer (1-3).

Para los Objetivos 4 y 5 se definen metas específicas a alcanzar el año 2015: disminuir la mortalidad infantil en menores de 5 años de edad en dos tercios (66\%) (ODM 4), y la mortalidad materna en tres cuartos (75\%) (ODM 5), comparadas con las tasas del año 1990. Los indicadores propuestos son: tasa mortalidad infantil (TMI) en menores de 5 años, razón de mortalidad materna (RMM), cobertura de atención profesional del parto y acceso universal a la salud materna y reproductiva (4-5). En la Tabla I, se presentan los objetivos e indicadores de acuerdo al tercer informe del Gobierno de Chile el año 2010 , que propone estrategias a realizar para el período 2011-2014 (6-7).

A nivel global se han logrado progresos importantes, reduciendo desde aproximadamente $500 \mathrm{mil}$ muertes maternas anuales en el año 1990 (RMM de $400 / 100.000 \mathrm{NV}$ ), a aproximadamente $287 \mathrm{mil}$ muertes en el año 2010 (210/100.000 NV) (rango: 170-300 muertes maternas/100.000 NV), es decir una disminución de más de un $40 \%$. Este período histórico de observación tan estricta ha permitido demostrar la enorme inequidad en la situación materna a nivel global y regional. Así la meta propuesta ha sido alcanzada sólo por una minoría de países, fundamentalmente de Europa oriental. En su gran mayoría (99\%), los países con peores indicadores han logrado a su vez progresos menores (1-5).

En nuestra región (Latinoamérica y el Caribe), se observa una tendencia sostenida a la mejoría de estos indicadores materno-infantiles, pero aún insuficiente para alcanzar las Metas del Milenio el 2015, particularmente cuando se analiza la tendencia desagregada a nivel de países (8-10). Nuestra región presenta una situación de inequidad inaceptablemente alta, la que se expresa por la persistencia de indicadores significativamente mayores en poblaciones con menores niveles de ingreso y educación, en poblaciones rurales y en pueblos indígenas.

\section{Realidad Materno-Infantil Nacional}

Chile ha presentado al igual que Uruguay, Cuba, Costa Rica y Méjico progresos significativos en el indicador de mortalidad materna, alcanzando porcentajes significativos de reducción en aproximadamente $50 \%$ en los últimos veinte años. Actualmente se encuentra entre los tres países con menor mortalidad materna del continente americano y el de menor mortalidad en toda Latinoamérica (9).

A nivel nacional al alcanzar indicadores de menos de 20 muertes maternas por cien mil nacidos vivos, el perfil etiológico ha cambiado de ser predominantemente de causas directas u obstétricas a causas indirectas asociadas al embarazo (8-10). 
Tabla I

OBJETIVOS MATERNO-INFANTILES DE DESARROLLO DEL MILENIO DE NACIONES UNIDAS. $(1990$ a 2015) (6-7)

Objetivo 4

Reducir la mortalidad de los niños menores de 5 años Meta (5)

Reducir en dos terceras partes, entre 1990 y 2015, la mortalidad de los niños menores de 5 años
Indicadores Mínimos

Tasa de Mortalidad de 1 a 4 años (por cada 1000 habitantes en edad respectiva).

Tasa de Mortalidad infantil (por cada 1000 nacidos vivos).

Porcentaje de niños de un año vacunados contra el sarampión

\section{Objetivo 5}

Mejorar la salud materna

Meta (6)

Reducir, entre 1990 y 2015, la tasa de mortalidad materna en tres cuartas partes

\section{Indicadores Mínimos}

Tasa de Mortalidad Materna (por 100.000 nacidos vivos) Porcentaje de partos con asistencia de personal sanitario especializado Indicadores adicionales

Uso de métodos anticonceptivos por mujeres de edad fértil (Proporción de mujeres de 15 a 49 años que usan algún método anticonceptivo, casadas o en relación de pareja)

Nacidos vivos de madres menores de 20 años sobre el total de nacidos vivos
La Figura 1 muestra el perfil por grandes causas de muerte para el año 2009 de acuerdo a estadísticas oficiales. Esta situación es concordante con el perfil epidemiológico materno actual, caracterizado por mayor edad de las madres y mayor frecuencia de enfermedades que coexisten con el embarazo (obesidad, hipertensión arterial, diabetes, hipotiroidismo), así como por la emergencia de mujeres que han prolongado su vida hasta alcanzar la edad fértil como resultado de avances logrados en el tratamiento de enfermedades crónicas o de resolución quirúrgica en la infancia y adolescencia (cardiopatías congénitas, nefropatías, trasplantes).

En la evaluación de un indicador tan sensible como el de mortalidad materna, se debe analizar además el grado de equidad en su distribución, así como también la tendencia observada. Estos dos últimos aspectos son dentro de una realidad materno-infantil aceptable, signos de alarma importantes para nuestro país: una inequidad persistente y un estancamiento de la situación materna en el último decenio.

\section{Desafíos Actuales}

Chile, fiel a la gran valoración social otorgada por su pueblo a la maternidad y a la niñez, concordante con su historia de priorizar políticamente los aspectos materno-infantiles, debe promover la

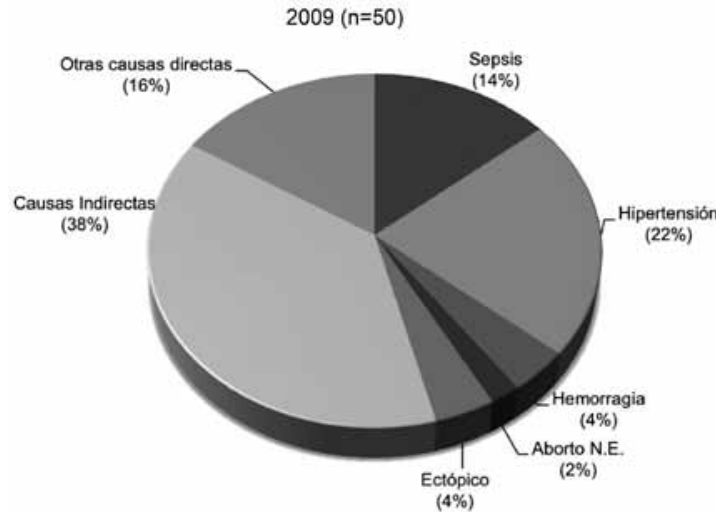

Figura 1. Causas de Mortalidad Materna. Chile 2009. (Fuente DEIS, Ministerio de Salud de Chile).

discusión de nuevas estrategias para mejorar esta situación. Las instituciones encargadas de ello deben promover la discusión al más alto nivel con el objetivo de generar nuevas políticas públicas orientadas a innovar y mejorar la situación en esta área. Parafraseando un editorial de la revista Lancet "todos sabemos lo que se debe hacer" (3), debemos reinstalar el tema y quebrar esta ausencia de progreso en la salud de nuestras madres. 
El perfil actual es complejo y el estancamiento de la mortalidad materna sugiere que se requiere una forma de abordaje nueva y complementaria a las grandes políticas públicas que le permitieron a Chile en los últimos 50 o más años, posicionarse como uno de los países del continente más seguros para ser madre (9).

La Sociedad Chilena de Obstetricia y Ginecología, el Ministerio de Salud y la Pontificia Universidad Católica de Chile, junto a la valiosa colaboración de expertos referentes nacionales e internacionales provenientes de Canadá y del Centro OMS-Rosarino de Estudios Perinatales, organizaron esta reunión de trabajo sobre mortalidad materna en nuestro país, realizada en Concepción, Chile, el 12 y 13 de Noviembre de 2012.

\section{Proposiciones de Consenso}

Existe concordancia en que por primera vez en los últimos cincuenta años, la situación materna está estancada, sin variaciones significativas en los últimos diez años (11). Esta situación es altamente inestable y por lo tanto es deseable que se implementen a corto plazo nuevos programas específicos orientados a enfrentar esta nueva situación epidemiológica. Así, este enfrentamiento debe incluir fundamentalmente dos ejes: en primer lugar, enfrentar la nueva realidad de salud de la mujer en edad fértil, reflejada en la ultima encuesta nacional de salud y el refuerzo en la seguridad de la asistencia del embarazo, parto y puerperio en los lugares más alejados y más vulnerables del país, donde nacen 12 mil niñas y niños anualmente, en condiciones que deben ser mejoradas.

Más allá de considerar una "falla" de abordaje de nuestras políticas públicas frente al estancamiento de la mortalidad materna, el patrón epidemiológico actual es extremadamente complejo y es importante notar que una meta prioritaria debe ser no sólo intentar reducir la mortalidad materna adicionalmente, sino también, impedir que ésta se incremente, como esta ocurriendo en varios países desarrollados. La actual situación sugiere que es necesario diseñar una estrategia para perfiles maternos de alto riesgo adicional, más acorde con el perfil demográfico y epidemiológico que presenta la nación en nuestros días.

Este documento pretende proponer desde nuestra experiencia positiva y con buenos resultados por todos conocidos. Es hacerse cargo responsablemente de la nueva realidad epidemiológica y promover al más alto nivel político el reforzamiento de las políticas públicas materno-infantiles con énfasis técnicos específicos logrados en este consenso.

\section{Reforzar los principales programas en curso en el área materno-infantil}

Se debe hacer un esfuerzo en reevaluar y reforzar la eficacia en la implementación de los principa- les programas actualmente en curso y correctamente implementados en esta área. Es fundamental el concepto de NO cambiar los aspectos fundamentales de nuestro modelo basado en niveles de atención y de derivación sino evaluar los aspectos fundamentales a mejorar y optimizar.

Las actividades realizadas por el Ministerio de Salud en el área materna y neonatal en los últimos dos años, se detallan en la Tabla II (información referida por coautores pertenecientes al Ministerio de Salud de Chile).

El rol de la matrona y/o matrón es fundamental en el manejo de la atención del nivel primario, porque son responsables del primer enfrentamiento en el diagnóstico y manejo en este nivel, es la base de nuestro sistema de control antenatal basado en niveles de complejidad. Estos profesionales también cumple roles cruciales en nuestro modelo de atención secundaria y terciaria que deben ser reforzados y que hacen que nuestro sistema tenga características positivas singulares que deben ser remarcados.

Reforzamiento de los programas actuales en términos de mayor acceso a la salud reproductiva, programas nutricionales y diagnóstico y apoyo del riesgo psicosocial detectado antes y durante el embarazo.

\section{Nuevas áreas de riesgo y proposiciones especí- ficas}

Priorizar aspectos que concentran el mayor riesgo de morbi-mortalidad materna con intervenciones basadas en la mejor evidencia científica disponible:

- Control preconcepcional orientado a diagnóstico y manejo de patologías prevalentes basado en la Encuesta Nacional de Salud, el concepto es la prevención mediante el manejo interdisciplinario y la educación.

- Reforzar rol de matronas y matrones, en los distintos niveles de atención.

- Control antenatal dirigido al diagnóstico de vulnerabilidad y riesgo psicosocial.

- Relocalizar la atención del parto en hospitales de baja complejidad.

- Racionalizar los recursos según situaciones de áreas geográficas específicas y niveles de atención.

- Reforzar el manejo y seguridad del día del parto. Ajustar los recursos según necesidades.

- Estrategias destinadas a disminuir las inducciones y cesáreas. Existe evidencia que el aumento de partos cesárea produce un aumento en la morbimortalidad materna. Existe evidencia que la mayoría de las cesáreas evitables provienen de inducciones innecesarias. Se debe realizar revisiones sistemáticas de las cesáreas realizadas, basadas en el cumplimiento de protocolos específicos.

- Evaluación del post parto y planificación familiar incluyendo orientación e información de las diferentes alternativas existentes (artificiales y naturales de efectividad y seguridad científicamente demostrada) en la actualidad. 
Tabla II

\section{ACTIVIDADES DESARROLLADAS POR EL MINISTERIO DE SALUD DE CHILE EN EL ÁREA MATERNA Y NEONATAL (2011-2012)}

Constitución de la Comisión Nacional de Obstetricia, Ginecología y Neonatología. Resolución Exenta Nº 10 de 11.04.11.

Formulación de la Estrategia Nacional de Salud 2011 - 2020: Se incorpora como meta de impacto la disminución de la mortalidad perinatal proyectada.

Constitución de la Comisión Nacional de Auditoría de Mortalidad Materna y Perinatal. Resolución Exenta $\mathrm{N}^{\circ} 1013$ de 16.12 .11$.

Actualización de la Norma № 100 y de los Formularios de Auditoría Materna, Fetal, Neonatal e Infantil.

Auditorias de muertes maternas y análisis de casos con antecedentes clínicos.

Elaboración de informes de mortalidad materna: análisis epidemiológico y recomendaciones.

Capacitación presencial y e-learning en la red asistencial: manejo de patología obstétrica, atención integral del recién nacido, emergencia obstétrica y neonatal.

Elaboración de documentos regulatorios: actualización guía perinatal; elaboración orientaciones técnicas para la atención de la puérpera y el recién nacido, manual de procedimientos para la atención del recién nacido en atención inmediata y puerperio, guía clínica de diabetes gestacional.

Actualización de guías clínicas: Parto Prematuro, Analgesia en el Parto.

Elaboración de un programa para evaluar servicios de obstetricia, ginecología y neonatología: aplicación de un piloto en 2013

Reforzar atención durante el posparto.

Elaboración de un protocolo para el manejo de la depresión en el embarazo y posparto.

Comisión nacional de transmisión vertical de VIH/Sífilis.

Mesas de trabajo elaboración de canastas por patología: Transfusión feto-fetal, infertilidad.

Participación en revisión y actualización de documento de redes GES y no GES.

Informatización del comprobante de parto.

Piloto de registro nacional de anomalías congénitas y seguridad de recién nacidos

Categorización riesgo dependencia en los servicios de gineco-obstetricia.

Elaboración de indicadores de calidad materno-infantil para la red asistencial.

Piloto BID-SRCel-MINSAL, identificación y biometría de recién nacidos, más estadísticas vitales en línea.

Modelamiento de procesos para la gestión asistencial hospitalaria.

Comisión Nacional de Lactancia Materna.

\section{Actividades específicas}

- Planificación específica, intervenciones y fines del control preconcepcional orientado al diagnóstico y manejo de las patologías de mayor prevalencia: obesidad, diabetes, hipertensión arterial y el hipotiroidismo, así como también la promoción de hábitos y estilos de vida saludables.

- Educación continua: cursos para profesionales de niveles primario, secundario y terciario. Protocolos específicos para los nuevos grupos de riesgo en aumento, las edades extremas de la vida reproductiva y el embarazo múltiple. 
- Página on line Ministerial con las guías de tratamiento de las patologías más prevalente y de riesgo, actualizadas para consulta permanente. Establecer línea de consulta de emergencia nacional permanente.

- Desarrollo a corto plazo de 10 a 15 guías clínicas específicas, actualizadas y basadas en la mejor evidencia disponible, de las patologías más importantes. Esto debe ser realizado por la Sociedad Chilena de Obstetricia, Universidades y Ministerio de Salud.

- Vigilancia estricta de los eventos fundamentales del día del parto. Especial orientación al manejo de la patología médica preexistente. Observatorio ejecutivo de los indicadores fundamentales, la información básica digital inmediata es fundamental.

- Racionalización de los sistemas de derivación y establecimientos de redes y coordinación de lo público y lo privado.

- Auditorías de la morbilidad severa (near miss) y de la mortalidad materna, con el fin de mejorar indicadores fundamentales.

- Reforzar y defender la importancia del uso de las auditorías de muerte materna como un instrumento exclusivamente clínico y epidemiológico y no como instrumento de penalización.

- Actividades preventivas: fomentar acceso a la salud reproductiva, facilitar elementos educativos para decisiones responsables. Particularmente, fortalecer los programas de consejerías en salud sexual de las adolescentes. Debemos considerar siempre como evitable la muerte materna de una adolescente.

- Comisión especial de estadísticas materno-infantiles. Compuesta por médicos, matronas y estadísticos capacitados en el tema. Informes anuales de los principales indicadores de impacto clínico adecuadamente escogidos.

- Mejorar y reforzar el registro de egresos hospitalarios utilizando la Codificación Internacional de Enfermedades (CIE) en uso a través de una norma técnica de los principales códigos por grandes grupos de causas del grupo $O$ (muertes maternas). Resolver ambigüedades a través de un consenso de expertos y comisión antes mencionada.

- Recomendar que un comité especial nacional (como la comisión perinatal ya creada) proponga un plan de implementación de las recomendaciones de este consenso. Esto debe incluir comités hospitalarios orientados a llevar al nivel local estas políticas.

- Abordar los determinantes sociales en la salud es crucial en lo esfuerzos para alcanzar mejores niveles de salud materna.

- Advocacía permanente en el alto nivel político sobre la prioridad de la salud materno-infantil. Trabajo conjunto de sociedades científicas con líderes femeninos de la sociedad.
Es deseable que este documento contribuya a un debate positivo orientado a mejorar la situación materna nacional, fieles a nuestra tradición de otorgar elevado valor social a la maternidad y la niñez. Las universidades, las sociedades profesionales y organizaciones comunitarias están disponibles para colaborar en conjunto con las instituciones gubernamentales en desarrollar e implementar nuevas iniciativas orientadas a mejorar la situación de las mujeres, madres y a través de ellas a sus hijas e hijos nacidos en nuestro país.

\section{REFERENCIAS}

1. Hogan MC, Foreman KJ, Naghavi M, Ahn SY, Wang M, Makela SM, et al. Maternal mortality for 181 countries, 1980-2008: a systematic analysis of progress towards Millennium Development Goal 5. Lancet 2010;375(9726):1609-23.

2. Waage J, Banerji R, Campbell O, Chirwa E, Collen$\operatorname{der} \mathrm{G}$, Dieltiens $\mathrm{V}$, et al. The Millennium Development Goals: a cross-sectoral analysis and principles for goal setting after 2015 Lancet and London International Development Centre Commission. Lancet 2010;376(9745):991-1023.

3. Campbell OM, Graham WJ; Lancet Maternal Survival Series steering group. Strategies for reducing maternal mortality: getting on with what Works. Lancet 2006;368(9543):1284-99.

4. WHO, UNICEF, UNFPA and The World Bank. Trends in Maternal Mortality: 1990 to 2008 . Estimates developed by WHO, UNICEF, UNFPA and The World Bank. World Health Organization 2010.

5. United Nations. The Millennium Development Goals Report 2011. Disponible en: http://www.un.org/millenniumgoals/11_MDG\%20Report_EN.pdf.

6. Objetivos de Desarrollo del Milenio: Tercer informe del Gobierno de Chile 2010. Disponible en: www.pnud. cl/odm/tercerinforme/Tercer\%20Informe\%20Nacional\%20ODM\%20Chile\%202010.pdf

7. Objetivos de Desarrollo del Milenio. Informe 2012. www.undp.org/content/dam/undp/library/MDG/spanish/MDG\%20Report\%202012\%20-\%20Complete\%20Spanish.pdf

8. Donoso E. Mortalidad Materna en Chile, 2000-2004. Rev Chil Obstet Ginecol 2006;71(4):246-51.

9. Koch E, Thorp J, Bravo M, Gatica S, Romero CX, Aguilera H, Ahlers I. Women's education level, maternal health facilities, abortion legislation and maternal deaths: a natural experiment in Chile from 1957 to 2007. PLoS One 2012;7(5):e36613.

10. González R. Salud Materno-Infantil en las Américas. Rev Chil Obstet Ginecol 2010;75(6):411-21.

11. Donoso E, Carvajal J. El cambio del perfil epidemiológico de la mortalidad materna en Chile dificultará el cumplimiento del $5^{\circ}$ objetivo del milenio Rev Med Chile;140:(10)1253-62. 